\title{
Nano-scale analyses of the chromatin decompaction induced by histone acetylation*
}

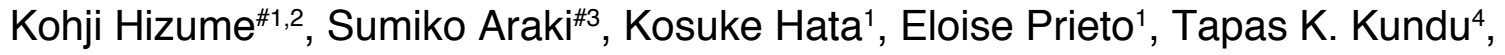 \\ Kenichi Yoshikawa ${ }^{3}$, and Kunio Takeyasu ${ }^{1}$
}

${ }^{1}$ Laboratory of Plasma Membrane and Nuclear Signaling, Graduate School of Biostudies, Kyoto University, Kyoto; ${ }^{2}$ Division of Microbial Genetics, National Institute of Genetics, Research Organization of Information and Systems, Mishima, Shizuoka; ${ }^{3}$ Department of Physics, Graduate School of Science, Kyoto University, Kyoto, Japan; and ${ }^{4}$ Transcription and Disease Laboratory, Molecular Biology and Genetics Unit, Jawaharlal Nehru Center for Advanced Scientific Research, Jakkur, PO Bangalore 560064, India

Summary. The acetylation of histone tails is a key factor in the maintenance of chromatin dynamics and cellular homeostasis. The hallmark of active chromatin is the hyper-acetylation of histones, which appears to result in a more open chromatin structure. Although short nucleosomal arrays have been studied, the structural dynamics of relatively long acetylated chromatin remain unclear. We have analyzed in detail the structure of

Received October 26, 2009

*This work was supported by the Japanese Ministry of Education, Culture, Sports, Science and Technology (Grantin-Aid for scientific Research on Priority Areas for K T [19207001] and K Y [17076007]), the Japan Society for the Promotion of Science (Grant-in-Aid for Young Scientists B for K H [21770167]), and by the Inamori Foundation. The DST (Government of India)-JSPS Collaborative Science Program (Bilateral Joint Projects) provided support to K T and T K. S A is a recipient of the JSPS (Japan Society for the Promotion of Science) predoctoral fellowship.

${ }^{\#} \mathrm{~K} \mathrm{H}$ and S A contributed equally.

Address for correspondence: Dr. Kohji Hizume, Division of Microbial Genetics, National Institute of Genetics, Research Organization of Information and Systems, 1111 Yata, Mishima, Shizuoka, 411-8540, Japan

Tel: +81-55-981-6757, Fax: +81-55-981-6762

Email:khizume@lab.nig.ac.jp long hyper-acetylated chromatin fibers using atomic force microscopy (AFM). Hyper-acetylated chromatin fibers isolated from nuclei that had been treated with Trichostatin A (TSA), an inhibitor of histone deacetylase, were found to be thinner than those from untreated nuclei. The acetylated chromatin fibers were more easily spread out of nuclei by high-salt treatment, implying that hyper-acetylation facilitates the release of chromatin fibers from compact heterochromatin regions. Chromatin fibers reconstituted in vitro from core histones and linker histone $\mathrm{H} 1$ became thinner upon acetylation. AFM imaging indicated that the gyration radius of the nucleosomal fiber increased after acetylation and that the hyper-acetylated nucleosomes did not aggregate at high salt concentrations, in contrast to the behavior of non-acetylated nucleosomal arrays, suggesting that acetylation increases long-range repulsions between nucleosomes. Based on these data, we considered a simple coarse grained model, which underlines the effect of remaining electric charges inside the chromatin fiber.

\section{Introduction}

In eukaryotic cells, genomic DNA is packed into a highly organized nucleoprotein complex (called chromatin). This complex is composed of nucleosomes, each of which consist of $147 \mathrm{bp}$ of DNA wrapped around a protein core made up of two copies each of the four core histone proteins (H3, H2B, H2A and H4) (Kornberg, 1974; Luger et al., 1997; Hizume et al., 2004). Nucleosomes give 
rise to a series of structures of increasing complexity. The simplest of these structures consists of an array of nucleosomes positioned in tandem on DNA in a "beadson-a-string" fiber that has a diameter of $11 \mathrm{~nm}$. This $11 \mathrm{~nm}$ fiber further condenses into the canonical " 30 nm fiber" with the help of linker histones (Thoma and Koller, 1977; Bustamante et al., 1997; Adkins et al., 2004; Hizume et al., 2005; Robinson and Rhodes, 2006). The $30 \mathrm{~nm}$ fiber forms loops and attaches to the nuclear matrix at semi-regular intervals along its length, most likely at every several tens- or hundreds-kbp (Hancock, 2000). This structure also further condenses into $\sim 100$ $\mathrm{nm}$ beaded fibers (Belmont and Bruce, 1994; Horn and Peterson, 2002).

Chromosome packing levels in the nucleus are believed to be closely linked to the degree of transcription. Genome regions with high transcriptional activity can be easily digested by DNase treatment (Weintraub and Groudine, 1976; Stalder et al., 1980). Electron microscopy studies show that RNA labeled with ${ }^{3} \mathrm{H}$-urysin is predominantly located in the less condensed region of the nuclei (Belyaeva and Zhimulev, 1976). These facts suggest that loosely packed genomic regions are active in transcription. It has also been reported that transcription is suppressed by histone $\mathrm{H} 1$ (Weintraub, 1984; O'Neill et al., 1995), which causes a compaction of the nucleosomal array into the $30 \mathrm{~nm}$ fiber. Taken together, these observations suggest that highly condensed "heterochromatin" regions lead to low transcriptional activities, while activated genes are located in the loosely packed "euchromatin" regions.

The chromatin state (heterochromatin or euchromatin) is dynamic and can be modulated by factors such as histone-tail modifications. Methylation of histone H3 lysine 9 induces interaction between the chromatin and heterochromatin protein 1 (HP1), which is involved in heterochromatin formation and gene silencing. Conversely, a positive correlation between histone acetylation and transcriptional activity was reported in 1964 (Allfrey et al., 1964). Since then, much research has supported the idea that histone acetylation leads to euchromatin formation (Kadonaga, 1998; Tse et al., 1998a; Zhang et al., 1998; Strahl and Allis, 2000), i. e., that the acetylation of histones leads to chromatin decondensation and thereby a looser chromatin structure. However, we are still far from completely understanding the effects that histone acetylation has on the chromatin structure from a global stance. Here, we have attempted to address the intriguing question of chromatin structurefunction using atomic force microscopy (AFM). We have carried out a nano-scale examination of chromatin dynamics in situ and in vitro and analyzed its dependence on the hyperacetylation of histones. Since we adopted a relatively large DNA chain of $26 \mathrm{kbp}$ for this study, it was possible to recognize the compacted chromatin structure as a three-dimensional fiber. The experimental system we used to reconstitute uniform fibers permitted consideration of chromatin fibers as a polymer. Since the components of chromatin are charged in solution, electrostatic interactions may govern chromatin structure. The acetylation of histone-tails alters the electrostatic charge of the histone, resulting in changes in the structural properties of the chromatin fiber. Measurements of the width of the chromatin fibers and nucleosome-nucleosome distances led us to consider a model of chromatin decondensation. These data suggested that changes in the total electrical charge of individual nucleosomes plays an important role in H1-induced 20-30 nm fiber formation and that the acetylation of histones significantly contributes to this alteration in charge.

\section{Materials and Methods}

\section{Cell culture, HDAC inhibitor treatment, and cell fractionation}

$\mathrm{HeLa} \mathrm{S} 3$ cells were cultured under $5 \% \mathrm{CO}_{2}$ at $37^{\circ} \mathrm{C}$ in complete Dulbecco's Modified Eagle's Medium (Sigma, St. Louis, MO, USA) supplemented with $10 \%$ fetal bovine serum (Hyclone, South Logan, UT, USA). To inhibit histone deacetylase, Trichostatin A (TSA; Wako, Osaka) or Sodium Butyrate (NaBu; Sigma) was added into the medium.

HeLa cells cultured on coverslips were washed twice with phosphate-buffered saline (PBS). The cells were treated with a nuclei isolation (NI) buffer $[100 \mathrm{mM} \mathrm{NaCl}$, $0.5 \%$ Triton $\mathrm{X}-100,3 \mathrm{mM} \mathrm{MgCl} 2,1 \mathrm{mM}$ EGTA, $10 \mathrm{mM}$ PIPES (pH 6.8)] at $4^{\circ} \mathrm{C}$ for $10 \mathrm{~min}$, and then with a high salt (HS) buffer $\left[250 \mathrm{mM}\left(\mathrm{NH}_{4}\right)_{2} \mathrm{SO}_{4}, 0.5 \%\right.$ Triton X-100, $3 \mathrm{mM} \mathrm{MgCl} 2,1 \mathrm{mM}$ EGTA, $10 \mathrm{mM}$ PIPES (pH 6.8)] at $25^{\circ} \mathrm{C}$ for $5 \mathrm{~min}$. Following fixation with PBS containing $4 \%$ paraformaldehyde at $25^{\circ} \mathrm{C}$ for $15 \mathrm{~min}$, each specimen was mounted on slides with Vectorshield containing DAPI.

For evaluation of the amount of fibers released from the nucleus, the intensity of DAPI staining outside the nucleus was measured using the Image J program. This intensity was then divided by the number of nuclei in the image, and the amount of released fibers was presented as the DAPI intensity of released fibers per nucleus (Fig. 1I). 

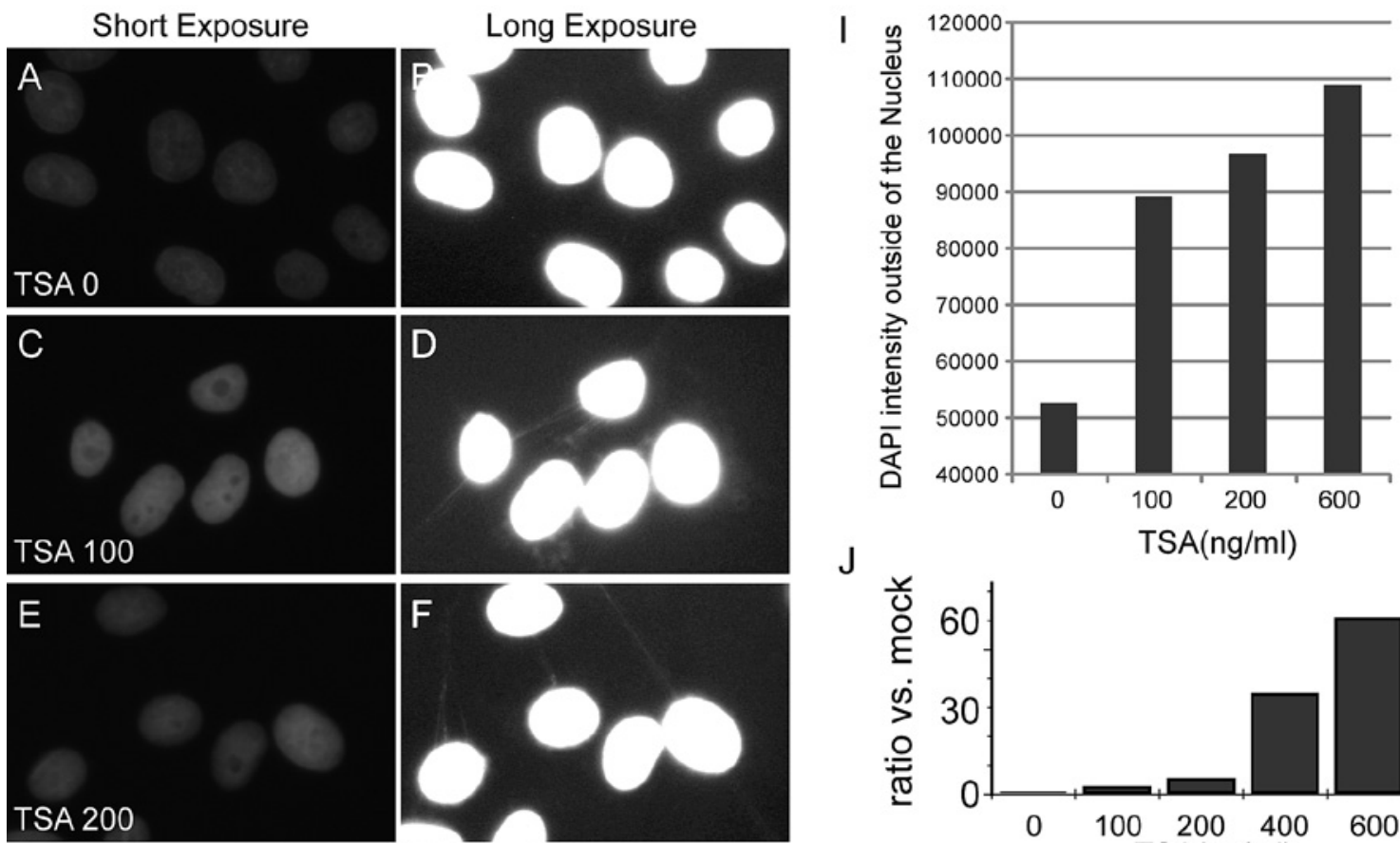

\section{$\mathrm{J}$}
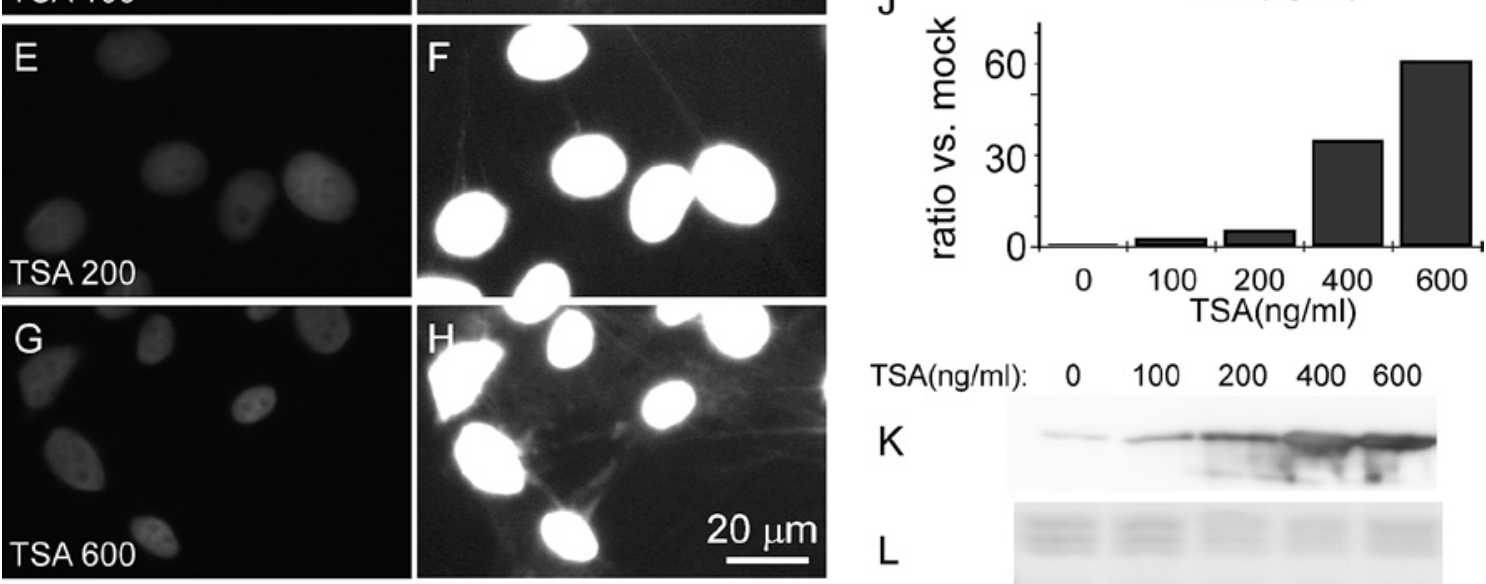

$\mathrm{TSA}(\mathrm{ng} / \mathrm{ml}): \quad 0 \quad 100 \quad 200 \quad 400 \quad 600$

$\mathrm{K}$

$\mathrm{L}$

Fig. 1. Treatment with an HDAC inhibitor induces chromatin fiber spread from nuclei. (A-H) DAPI stained nuclei isolated from non-treated cultured cells $(\mathbf{A}, \mathbf{B})$ or from cells treated with $100 \mathrm{ng} / \mathrm{m} l(\mathbf{C}, \mathbf{D}), 200 \mathrm{ng} / \mathrm{m} l(\mathbf{E}, \mathbf{F})$ or $600 \mathrm{ng} / \mathrm{ml}(\mathbf{G}, \mathbf{H})$ of trichostatin A for $24 \mathrm{~h}$. For each condition, the same field was captured using a short (A, C, E, G) and a long $(\mathbf{B}, \mathbf{D}, \mathbf{F}, \mathbf{H})$ exposure time. The longer time allowed the careful detection of spread fibers although nuclear DAPI signals were saturated. I: DAPI intensity in the area outside the nucleus was measured to estimate the degree of fiber spread and was compiled as a histogram. J-L: Detection of the level of acetylation of histone H3 by Western blotting. Cells treated with the indicated concentrations of TSA were harvested and cell extracts were loaded onto an SDS-poly-acrylamide gel, transferred to a membrane, and analyzed by Western blotting using an antibody against acetylated H3 (K). The same extracts were analyzed by a second SDS-poly-acrylamide gel electrophoresis followed by staining of the proteins with Coomassie Brilliant Blue (CBB). Panel $\mathbf{L}$ shows subunits of core histones stained with $\mathrm{CBB}$ that were used as loading controls. The intensities of the bands in the Western blotting shown in panel $\mathbf{K}$ were measured and compiled into a histogram $(\mathbf{J})$. The $\mathrm{Y}$ axis represents the value of the intensity relative to that of non-treated cells that was assigned a value of one.

\section{Isolation and lysis of nuclei and fluorescence observation}

HeLa cells, collected using a cell scraper, were washed twice with phosphate-buffered saline (PBS). The cells were treated with a NI buffer at $4^{\circ} \mathrm{C}$ for $10 \mathrm{~min}$. The nuclei were collected after centrifugation $(1,000 \times \mathrm{g}$ for $5 \mathrm{~min}$ ) and resuspended in a NI buffer without Triton $\mathrm{X}-100$.

The nuclear suspension was dropped onto a coverslip that had been pre-treated with $0.02 \%$ poly-L-lysine at $25^{\circ} \mathrm{C}$ for $15 \mathrm{~min}$. The surface of the coverslip was dried 
once to absorb the nuclei, treated with a HS buffer at $25^{\circ} \mathrm{C}$ for $5 \mathrm{~min}$, and then fixed with PBS containing $0.5 \mu \mathrm{g} / \mathrm{m} l$ DAPI and $4 \%$ paraformaldehyde at $25^{\circ} \mathrm{C}$ for $15 \mathrm{~min}$.

To stain lipid membranes, the nuclei mounted on glass coverslips were treated with $\mathrm{DiOC}_{5}$ (1:5,000 dilution; Invitrogen, Carlsbad, CA, USA). To stain the nuclear pore complex (NPC) or lamin B, the nuclei specimens were treated with specific primary antibodies (anti-NPC monoclonal antibody; Berkeley Antibody Company, Richmond, CA, USA, or anti-lamin B antibody; Santa Cruz Biotechnology, Inc., Santa Cruz, CA, USA) and fluorescent secondary antibodies (anti-mouse IgG antibody conjugated with FITC; Invitrogen, or antigoat IgG antibody conjugated with Alexa Fluor 488; MP Biomedicals, Irvine, CA, USA). Fluorescently stained nuclei were observed using a confocal fluorescence microscope (LSM5 Pascal, Zeiss, Germany) in Figure 2.

\section{Plasmid DNA}

The plasmid used for chromatin reconstitution was a kind gift from Dr. W. De Laat (Erasmus University Medical Center, Department of Cell Biology, the Netherlands). The locus control region (LCR) of the human $\beta$-globin gene $(21.5 \mathrm{~kb})$ was isolated by Sal I/Cla I digestion and subcloned into pBR322, yielding the $26 \mathrm{~kb}$ plasmid.

\section{Histones}

Core histones were purified by a previously described procedure (Hizume et al., 2005, 2007). Briefly, nuclei were isolated by low-speed centrifugation from HeLa cells, which had been treated with a buffer containing $0.5 \%$ Triton $\mathrm{X}-100$. The nuclei were then treated with micrococcal nuclease (40 units/mg of DNA) at $37^{\circ} \mathrm{C}$ for $15 \mathrm{~min}$, and solubilized fractions containing monoor oligo-nucleosomes were collected. Core histones were separated from DNA by hydroxyapatite column chromatography (Simon and Felsenfeld, 1979), and the purified core histones were applied to a gel filtration column (HiPrep 16/60 S-200, GE Healthcare UK Ltd., Buckinghamshire, England) to separate the octamer from the $\mathrm{H} 3-\mathrm{H} 4$ tetramer, the $\mathrm{H} 2 \mathrm{~A}-\mathrm{H} 2 \mathrm{~B}$ dimer, and other contaminants. Histone H1 was obtained from Calbiochem.

The p300 acetyltransferase (p300), which was used for histone-tail acetylation, was purified from recombinant baculovirus-infected Sf 21 cells as a His6-tagged protein using a nickel-nitrilotriacetic acid affinity column (Qiagen, Hilden, Germany), as described previously (Balasubramanyam et al., 2003).

To obtain acetylated histones, purified core histones were dialyzed against a buffer containing $50 \mathrm{mM}$ Tris-
$\mathrm{HCl}(\mathrm{pH} 8.0), 10 \%$ glycerol, $1 \mathrm{mM}$ dithiothreitol, $1 \mathrm{mM}$ PMSF, $0.1 \mathrm{mM}$ EDTA(pH 8.0), and $10 \mathrm{mM} \mathrm{NaBu}$ at $44^{\circ} \mathrm{C}$ for $24 \mathrm{~h}$. The dialyzed core histones $(16 \mu \mathrm{g})$ were treated with p300 (60 ng) and acetyl-CoA (56 $\mu \mathrm{M})$ (Sigma) at $36^{\circ} \mathrm{C}$ for $7 \mathrm{~h}$. As a non-acetylated control, the dialyzed core histones were treated with acetyl-CoA only.

Acetylation levels of histones were analyzed by gel electrophoresis using an AUT gel, as described elsewhere (Zweidler, 1978). The acetylated histones were incubated with an equivalent volume of a loading buffer [ $8 \mathrm{M}$ urea, $0.2 \%$ Pyronin Y, $1.4 \mathrm{mM} 2$-mercaptoethanol] for $5 \mathrm{~min}$. The mixture was applied onto a gel containing 5\% acetic acid, $8 \mathrm{M}$ urea, $12 \%$ acrylamide, and $0.4 \%$ Triton X-100 and electrophoresed in $0.9 \mathrm{~N}$ acetic acid. Proteins were stained using a silver staining kit (Wako).

\section{Chromatin reconstitution}

Equal amounts $(0.5 \mu \mathrm{g})$ of purified DNA and the histone octamer were mixed in a Hi-buffer $[10 \mathrm{mM}$ Tris- $\mathrm{Cl}(\mathrm{pH}$ 7.5), $2 \mathrm{M} \mathrm{NaCl}, 1 \mathrm{mM}$ EDTA, 0.05\% NP-40, $5 \mathrm{mM}$ $\beta$-mercaptoethanol, and $0.1 \mathrm{mM}$ PMSF] and placed in a dialysis tube (total volume, $50 \mu l$ ). Dialysis was begun by stirring the tube in $150 \mathrm{~m} l$ of the Hi-buffer at $4{ }^{\circ} \mathrm{C}$. A Lo-buffer [10 mM Tris-Cl (pH 7.5), 1 mM EDTA, 0.05\% NP-40, $5 \mathrm{mM}$ 2-mercaptoethanol, and 0.1 mM PMSF] was then added to the dialysis buffer at a rate of $0.46 \mathrm{~m} / /$ min, and, simultaneously, the existing dialysis buffer was pumped out at the same speed with a peristaltic pump so that the final dialysis buffer contained $50 \mathrm{mM} \mathrm{NaCl}$ after $20 \mathrm{~h}$. The samples were collected from the dialysis tube and stored at $4^{\circ} \mathrm{C}$ until further use.

For reconstitution with histone $\mathrm{H} 1$, this was added after the salt dialysis was completed (at a $\mathrm{NaCl}$ concentration of $50 \mathrm{mM}$ ). The molar ratio of histone $\mathrm{H} 1$ to the histone octamer was 1:1 unless otherwise stated.

For AFM imaging, the reconstituted chromatin solution was fixed with $0.3 \%$ glutaraldehyde for $30 \mathrm{~min}$ at room temperature. The fixed sample was dropped onto a freshly cleaved mica surface that had been pretreated with $10 \mathrm{mM}$ spermidine. After 10 min incubation at room temperature, the mica was washed with water and dried under nitrogen gas.

\section{AFM observation}

AFM observations of the reconstituted chromatin and isolated nuclei were performed in air with both a Multi Mode and Bioscope II (Digital Instruments, Santa Barbara, CA, USA), using the tapping mode. The cantilever (OMCL-AC160TS-W2, Olympus, Tokyo) used was $129 \mu \mathrm{m}$ in length with a spring constant of 
33-62 N/m. The scanning frequency was $2-3 \mathrm{~Hz}$, and images were captured with the height mode in a $512 \times 512$ pixel format. The obtained images were plane-fitted and flattened by the computer program supplied in the imaging module to remove the background curvature of the mica surface.

The widths of chromatin fibers in AFM images were affected by the size of the tips used, and the apparent dimensions of the molecules obtained by AFM were dependent upon the radius of the tip curvature and appeared larger than the actual dimensions. To prevent this broadening effect caused by the tip ("tip effect") for the reconstituted chromatin fiber shown in Figure 4, the apparent size of the nucleosomes was used as a reference. This method is the same as that used in our previous reports concerning reconstituted chromatin (Hizume et al., 2004, 2005). The relationship between the width of the globular molecule in the AFM image $(W)$, the radius of the tip curvature $\left(R_{c}\right)$, and the radius of the molecule $\left(R_{m}\right)$, is given by $W=4\left(R_{c} \mathrm{R}_{m}\right)^{1 / 2}$. When two different molecules with $R_{m 1}$ and $R_{m 2}$ radii were imaged with the same tip, the relationship between the measured widths $\left(W_{l}\right.$ and $W_{2}$ ) was given by $W_{l}=W_{2}\left(R_{m l} / R_{m 2}\right)^{1 / 2}$. Since the diameter of the nucleosome is known to be $11 \mathrm{~nm}$ (Luger et al., 1997), we used the diameter of nucleosomes in our AFM images as $W_{1}$, and calculated the radius of the fiber $\left(R_{m 2}\right)$ from the apparent width of the fiber $\left(W_{2}\right)$.

To measure the width of the fiber spread from nuclei (Fig. 3), the fiber widths were estimated at the halfmaximum height (FWHM: full width at half-maximum). This method of measuring fiber width was previously used in our studies of genome fiber spread from cells (Kim et al., 2004; Kobori et al., 2006, 2007). This measurement is expected to subtract the broadening effect of the tip as described previously (Schneider et al., 1998) without the need for any size references.

\section{Gyration radius}

The positions of the nucleosomes were digitized as. $\vec{r}_{r}$ The radius of gyration $\left(R_{g}{ }^{2}\right)$ was calculated as follows:

$$
R_{g}{ }^{2}=\sum_{i=1}^{N}\left(\vec{r}_{i}-\vec{r}_{G}\right)^{2} / N
$$

where $\vec{r}_{G}$ is the center of mass.

\section{Results}

\section{TSA treatment affects chromatin packing}

To analyze the role of histone acetylation on chromatin fiber structure, we first assayed the effect of the inhibition of histone deacetylase on chromatin fiber spread from the cell nuclei. HeLa cells, cultured on coverglasses, were therefore treated with various concentrations of the histone deacetylase inhibitor TSA, and chromatin fibers were microscopically analyzed. For this purpose, the cells were treated with detergent to remove the plasma membrane as well as with cytosol $(0.5 \%$ Triton $\mathrm{X}-100$, $10 \mathrm{~min})$ followed by a high-salt $\left(250 \mathrm{mM}\left(\mathrm{NH}_{4}\right)_{2} \mathrm{SO}_{4}\right)$ treatment to remove nucleoplasmic proteins. Nucleic acids were then stained by DAPI (Fig. 1). During this lysis procedure, there was no spread of chromatin fiber out of nuclei which had not been treated with TSA (Fig. 1A, B). Therefore, the genome structures of control cells were tightly held inside the nucleus and remained inside the nuclei even after $\left(\mathrm{NH}_{4}\right)_{2} \mathrm{SO}_{4}$ treatment to remove the nucleoplasm. Treatment with high concentrations of TSA induced the appearance of more fibrous structures that did spread out of the nuclei (Fig. 1C-H). The degree of fiber release from the isolated nuclei was estimated based on the intensity of DAPI staining outside the nucleus (see Materials and Methods for details). As the concentration of TSA increased, there was a corresponding increase in the number of chromatin fibers that spread out of the nuclei. Western blotting analysis of acetylated histone H3 (Fig. 1K) indicated that the level of histone acetylation increased in tandem with the TSA concentration used and also correlated with the increased DAPIstaining of the spread-out fibers (Fig. 1I). Presumably, upon hyperacetylation due to the inhibition of histone deacetylases, the chromatin fibers decondensed, detached from the nuclear membrane scaffold, and were therefore more easily released from the nucleus.

\section{Nuclear isolation and AFM observation of TSA treated cells}

To analyze the structure of the chromatin fibers that spread from the nucleus following TSA treatment, AFM analysis was performed on isolated nuclei. HeLa nuclei were isolated by gentle centrifugation $(1000 \times \mathrm{g}, 5 \mathrm{~min})$ following detergent treatment $(0.5 \%$ Triton $\mathrm{X}-100,10$ min), and were then deposited on a glass surface (Fig. 2A). At this stage, the nuclear envelope was still intact, as shown by staining with DiOC6-which recognizes lipid membranes (Fig. 2B) - and by staining with anti-NPC (nuclear pore complex) (Fig. 2C) and anti-nuclear lamina 
A

\section{Culture HeLa cells \\ $\downarrow$}

Isolate nuclei from the cells

(Wash with $0.5 \%$ Triton X-100)

$\downarrow$

Mount the nuclei on glass surface

$\downarrow$

Lysis the nuclei with $250 \mathrm{mM}\left(\mathrm{NH}_{4}\right)_{2} \mathrm{SO}_{4}$
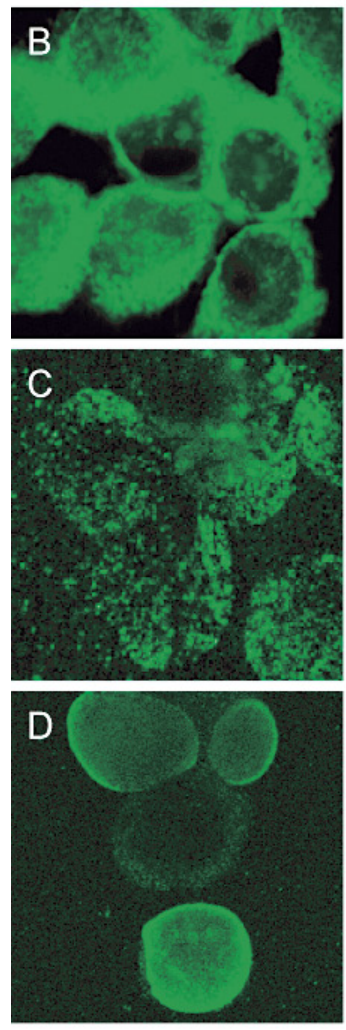
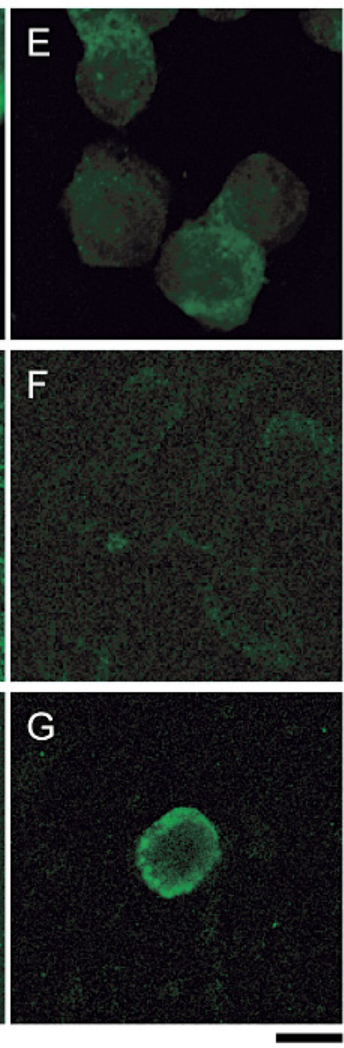
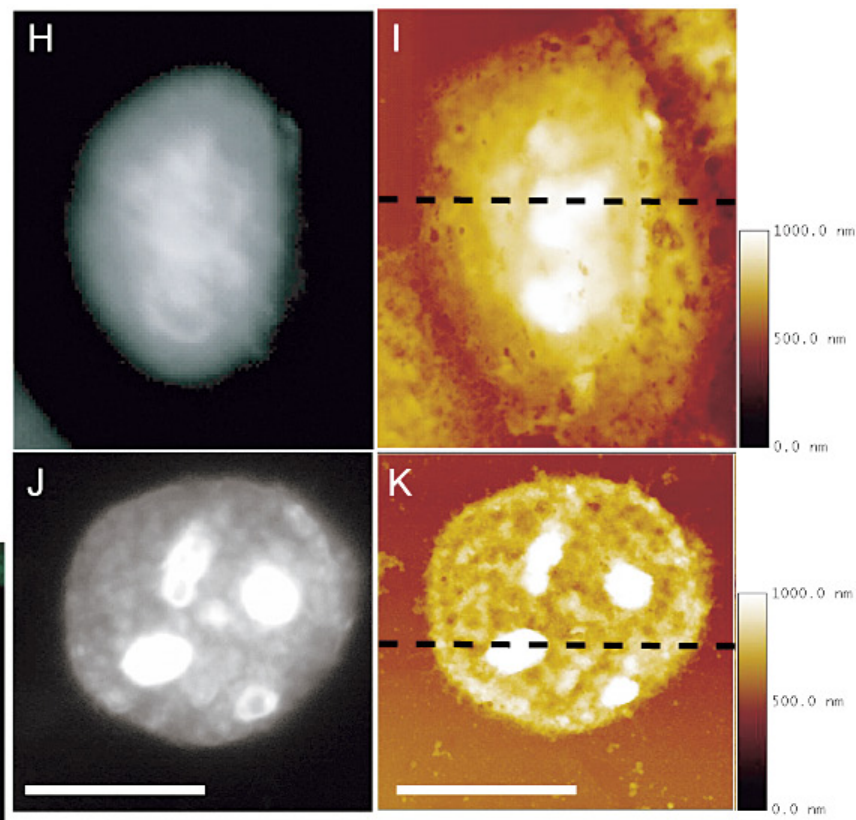

$\mathrm{L}$

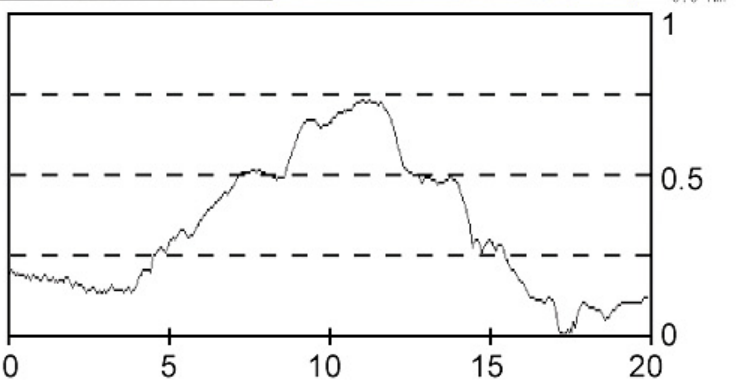

$\mathrm{M}$

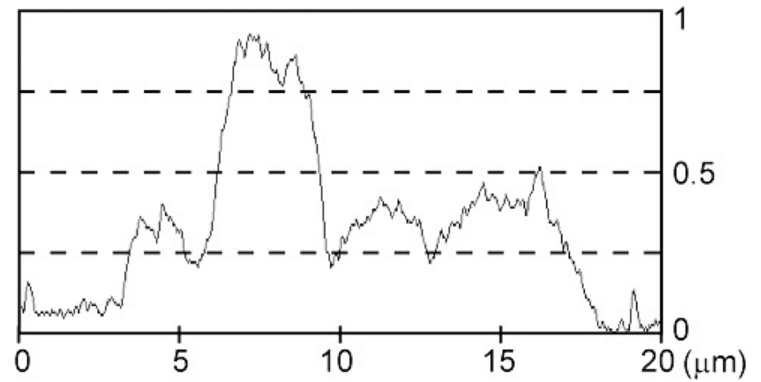

Fig. 2. Fluorescent analysis of nuclei isolated from HeLa cells by high salt-lysis on a glass surface. A: A brief outline of the procedure for nuclei isolation is shown. Cultured HeLa cells were harvested and treated with a buffer containing $0.5 \%$ Triton $\mathrm{X}-100$. Nuclei were isolated by low speed centrifugation, mounted on a glass surface, and lysed with a buffer containing $250 \mathrm{mM}\left(\mathrm{NH}_{4}\right)_{2} \mathrm{SO}_{4}$. B-G: Isolated nuclei before $(\mathbf{B}-\mathbf{D})$, and after $(\mathbf{E}-\mathbf{G}),\left(\mathrm{NH}_{4}\right)_{2} \mathrm{SO}_{4}$ treatment were observed by fluorescent microscopy following staining with DiOC6 - which selectively labels lipid membranes (B and E) - or following immunostaining with the anti-NPC (C and F), or anti-laminB (D and $\mathbf{G})$ antibodies. Prior to high salt lysis, the DiOC6 fluorescent signal was clearly detected both on the edge of and outside the nuclei, indicating that both the nuclear membrane and the ER were intact. The isolated nuclei were also observed to contain both NPC (C) and lamin (D) prior to lysis. H, J: DAPI-stained images of an isolated nucleus before (H), and after (J), high salt-lysis. I, K: AFM images of the same nuclei shown in $\mathrm{H}$ and $\mathbf{J}$ respectively. The dotted lines in (I) and (K) represent the sites at which the height profiles shown in panels $\mathbf{L}$ and $\mathbf{M}$ were respectively taken. Bars $=10 \mu \mathrm{m}$ 

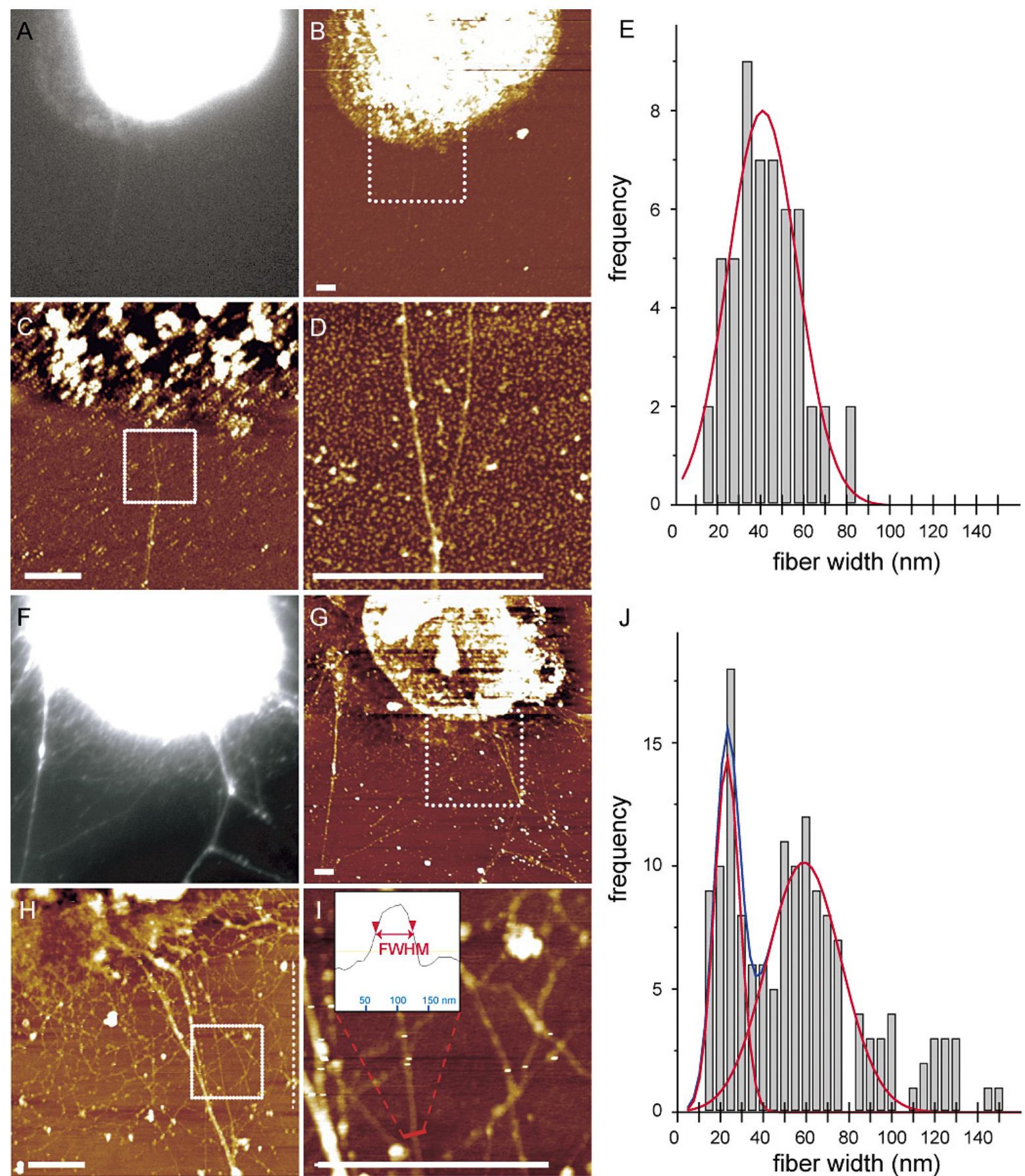

$\mathrm{J}$

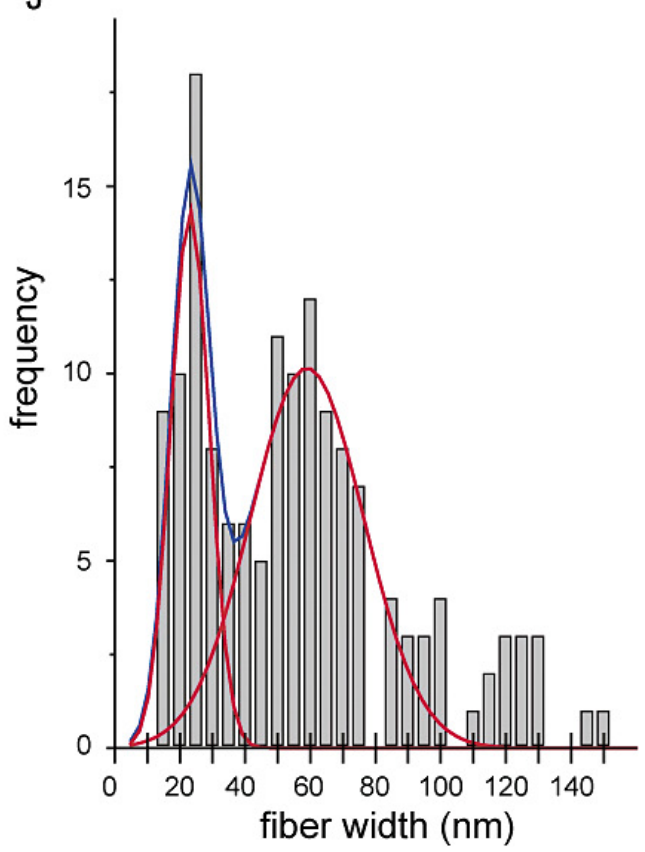

Fig. 3. AFM analysis of chromatin fibers spread out from nuclei. Nuclei were isolated from HeLa cells grown in the absence of treatment (A-E), or following treatment with $600 \mathrm{ng} / \mathrm{m} l \mathrm{TSA}$ and $10 \mathrm{mM} \mathrm{NaBu}$ for $24 \mathrm{~h}(\mathbf{F}-\mathbf{J})$. The nuclei were mounted on a glass surface, lysed with high-salt, and then the same region of the slide was observed under a fluorescence microscope following DAPI staining $(\mathbf{A}, \mathbf{F})$ and by AFM $(\mathbf{B}-\mathbf{D}, \mathbf{G}-\mathbf{I})$. Rescanning of the area within the dotted square in panels $\mathbf{B}$ and $\mathbf{C}$ is shown in panels $\mathbf{C}$ and $\mathbf{D}$, respectively. Rescanning of the dotted square in panels $\mathbf{G}$ and $\mathbf{H}$ is shown in panels $\mathbf{H}$ and $\mathbf{I}$, respectively. The width of the fibers shown in panels $\mathbf{D}$ and $\mathbf{I}$ were measured and compiled into a histogram ( $\mathbf{E}$ and $\mathbf{J}$ respectively). Cross-sections of the fibers (as shown in the inset of panel $\mathbf{I}$ ) were analyzed to measure FWHM (see materials and methods). Cross-sections of 53 areas of 13 fibers in panel D, and of 147 areas of 21 fibers in panel $\mathbf{I}$, were analyzed. Bars $=2 \mu \mathrm{m}$ 
A

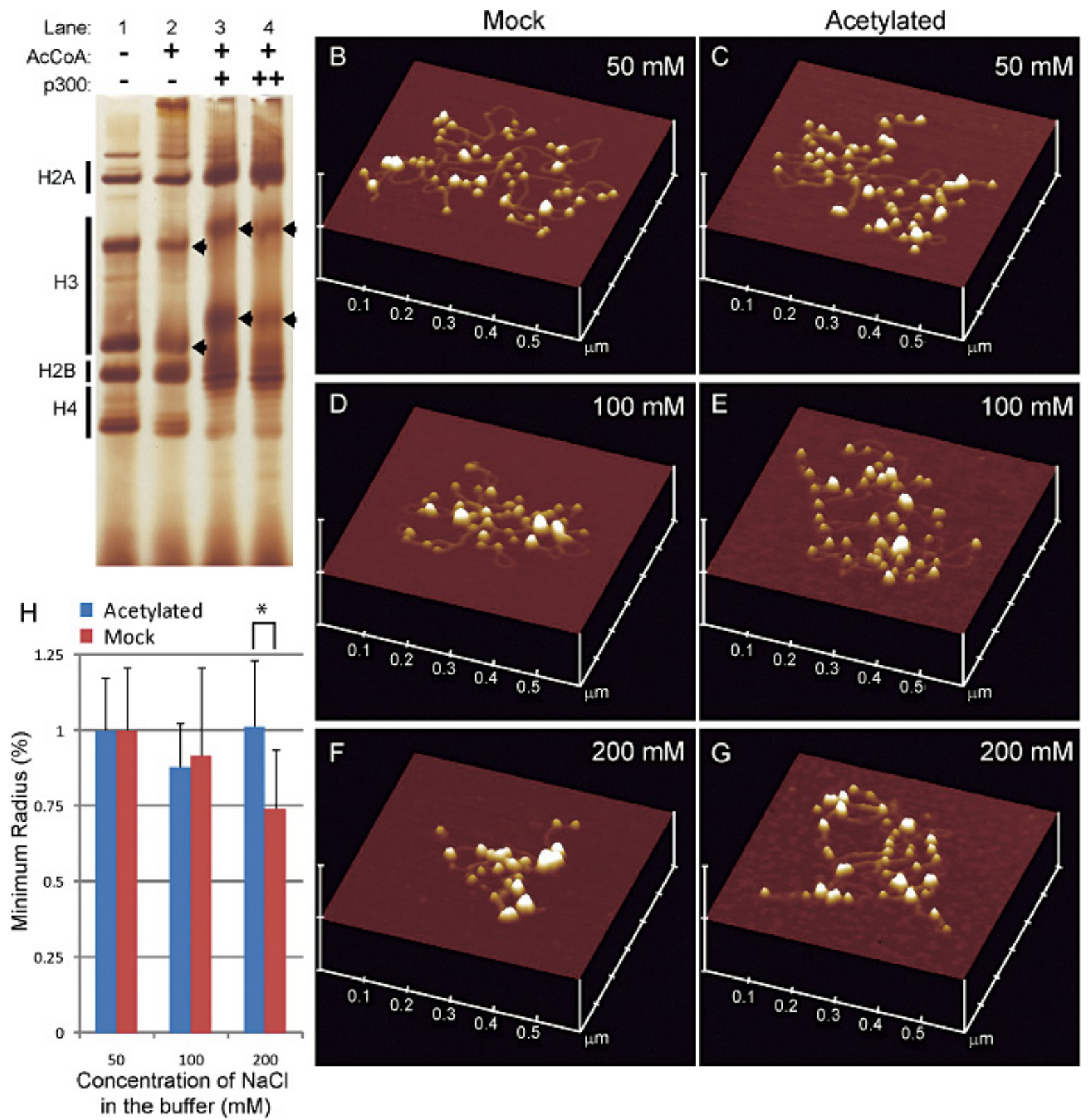

Fig. 4. Nucleosome reconstitution using acetylated core histones. A: Core histones were treated with or without acetylCoA and p300 and were then fractionated by Triton- acrylamide -urea gel electrophoresis. The histones were detected by silver-staining. The mobility of the core histone subunits was retarded with acetylation (arrows). B, D, F: An AFM image of nucleosomes reconstituted on a 26-kb plasmid using non-acetylated core histones (Mock). Nucleosomes were fixed in a buffer containing 50 (B), 100 (D) or $200 \mathrm{mM}$ (F) NaCl. C, E, G: An AFM image of nucleosomes reconstituted on a 26-kb plasmid using acetylated core histones. Nucleosomes were fixed in a buffer containing $50(\mathbf{C}), 100(\mathbf{E})$ or $200 \mathrm{mM}$ (G) $\mathrm{NaCl}$. H: The average minimum radius required for a circle that would enclose an entire chromatin molecule was measured in AFM images such as those shown in panels B-G. In the $200 \mathrm{mM} \mathrm{NaCl}$ buffer, the minimum radius of the non-acetylated nucleosomes was significantly smaller $\left({ }^{*} p=0.0014\right)$ than that of the acetylated nucleosomes. 

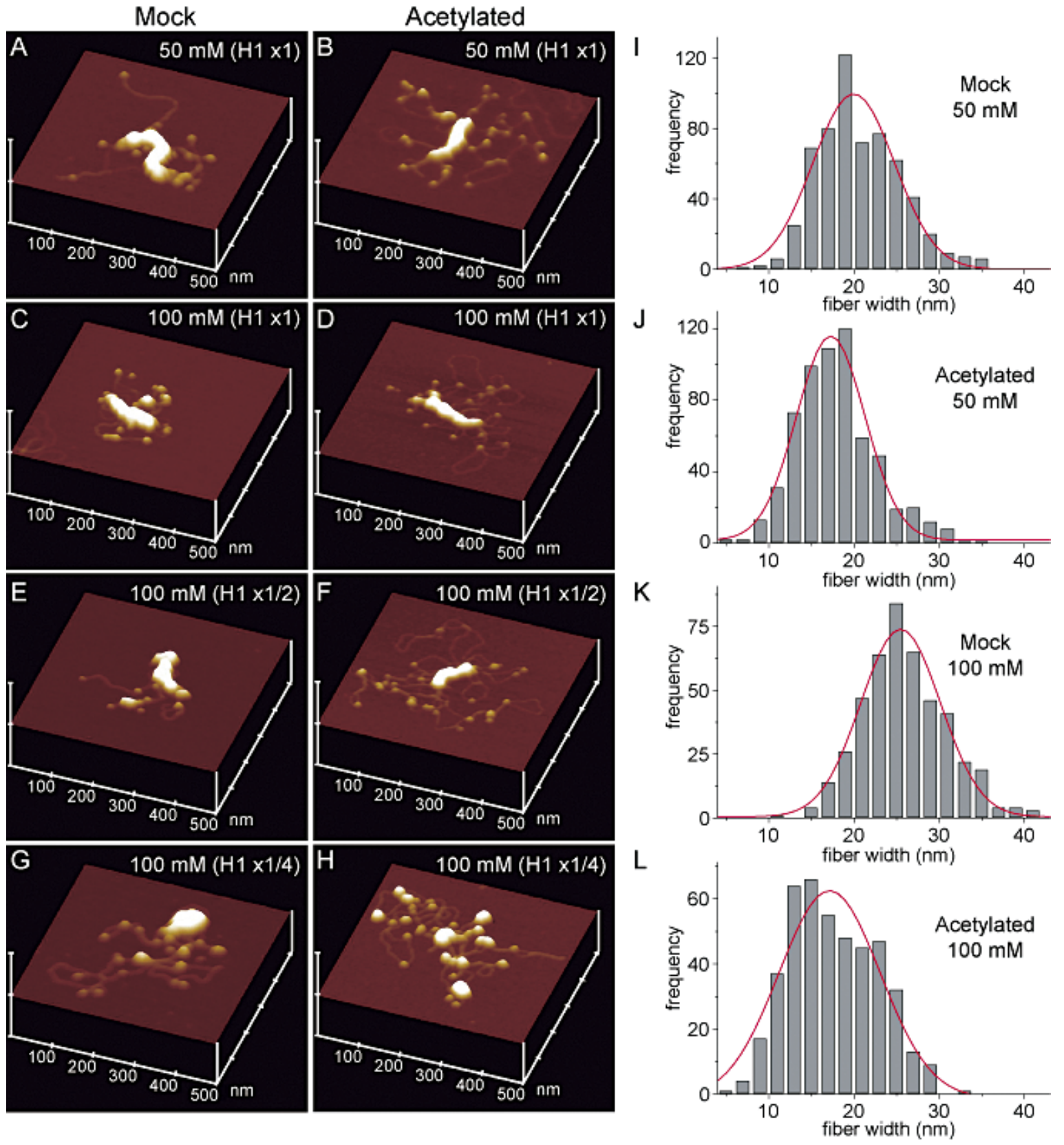

Fig. 5. An $\mathrm{H} 1$ induced fiber reconstituted on a $26 \mathrm{~kb}$ plasmid using non-acetylated or acetylated core histones. A-H: AFM images of H1-induced chromatin fibers reconstituted on a 26-kb plasmid using non-acetylated core histones (A, $\mathbf{C}, \mathbf{E}, \mathbf{G})$ or acetylated core histones $(\mathbf{B}, \mathbf{D}, \mathbf{F}, \mathbf{H})$. The samples were fixed in a buffer containing $50 \mathrm{mM}(\mathbf{A}$ and $\mathbf{B})$ or $100 \mathrm{mM}(\mathbf{C}-\mathbf{H}) \mathrm{NaCl}$. Histone $\mathrm{H} 1$ and the core histone octamer were added at a molar ratio of 1:1 (A-D), 1:2 (E and F) or 1:4 $(\mathbf{G}$ and $\mathbf{H})$. At a molar ratio of 1:1 of core histone octamer to linker histone H1, the lengths of the fibers were $207 \mathrm{~nm}$ and $224 \mathrm{~nm}$ in acetylated and non-acetylated chromatin, respectively. I-L: The widths of the fibers shown in panels A, B, C and $\mathbf{D}$ were measured and compiled into a histogram. Cross-sections of 644 areas of 20 fibers $(\mathbf{E}), 568$ areas of 25 fibers (G), 444 areas of 10 fibers (I) and 439 areas of 21 fibers (K) were analyzed to measure the bottom-tobottom distance and to remove the 'tip effect' as described in Materials and Methods. 
(Fig. 2D) antibodies. Subsequently, the nuclei were treated with $250 \mathrm{mM}\left(\mathrm{NH}_{4}\right)_{2} \mathrm{SO}_{4}$ to remove nucleoplasmic proteins. The DiOC6 and NPC-fluorescence signals could not be detected after this treatment (Figs. 2E and F, respectively). Although the nuclear lamina-fluorescence signal could still be detected (Fig. 2G), it was much weaker than before the treatment. Examination of the nucleus by DAPI staining revealed a distinct roundshaped nucleus prior to $\left(\mathrm{NH}_{4}\right)_{2} \mathrm{SO}_{4}$ treatment (Fig. $2 \mathrm{H}$ ). However, the outline of the nucleus was difficult to identify by AFM imaging (Fig. 2I), and AFM images showed a smooth nuclear surface (Fig. 2L), possibly due to the remaining ER surrounding the nucleus. Following $\left(\mathrm{NH}_{4}\right)_{2} \mathrm{SO}_{4}$ treatment, the outline of the nucleus was clearly identified by both DAPI-staining (Fig. 2J) and AFM imaging (Fig. 2K). It is likely that this treatment removed the nuclear envelope (Fig. 2E), resulting in a decrease in the height of the AFM image, and the appearance of a rough surface (Fig. 2M).

Few chromatin fibers were detected outside of the nuclei in the absence of TSA treatment, indicating that chromatin fibers were still tightly packed even after removal of the nuclear envelope (Fig. 3A-D). The fibers that were observed by AFM imaging were calculated to have a width of $43.0 \pm 15.6 \mathrm{~nm}$ (mean \pm S.D., $\mathrm{n}=53$; Fig. 3E), which is in agreement with our previous work (Kobori et al., 2006). In contrast, the AFM images of TSA-treated-nuclei revealed many fiber bundles of various widths (Fig. 3E-J). The thinnest fibers appeared to co-exist in bundles. Interestingly, the width of the thinnest fibers was measured as $23.1 \pm 5.9 \mathrm{~nm}$ (left peak of Fig. 3J), which is thinner than the fibers of the nonTSA-treated nuclei.

\section{Acetylated nucleosomes form fiber structures in vitro in the presence of the linker histone $\mathrm{H} 1$ that are thinner than those formed in non-acetylated nucleosomes}

In order to confirm the effect of histone acetylation on chromatin fiber structure, we performed AFM studies using an in vitro reconstituted chromatin fiber. For this purpose, highly purified HeLa core histones were acetylated with histone-acetyltransferase (p300). The results of the Acid-Urea-Triton (AUT) gel electrophoresis shown in Figure 4A confirm that subunits of core histones treated with p300 and acetyl-CoA exhibit decreased in mobility (lane 3) compared with non-treated core histones (lane1) or with core histones treated with only acetylCoA (lane 2). This suggests that the positive charges of the core histones subunits were decreased by acetylation; it is consistent with the expectation that the acetylation of lysine residues will neutralize some of the positive charge of histones. Excess treatment with p300 and acetylCoA did not further change the gel mobility of histones (lane 4), suggesting that the core histones in lane 3 were acetylated at all p300 targets within the protein.

AFM images of reconstituted chromatin, formed by the assembly of a $26-\mathrm{kb}$ plasmid DNA and core histones treated with only acetyl-CoA (non-acetylated core histones, Mock) or with acetyl-CoA and p300 (acetylated core histones), are shown in Figures 4B and $\mathrm{C}$ respectively. The chromatin reconstituted with non-acetylated core histones contained $55 \pm 5.0 \quad(n=8)$ nucleosomes, and that reconstituted with acetylated core histones contained $52 \pm 7.9$ nucleosomes per molecule of $26-\mathrm{kb}$ plasmid, indicating that histone-tail acetylation did not affect the efficiency of nucleosome formation as such. Interestingly, the distance between nucleosomes was affected by acetylation of the histones. Measurement of the gyration radius of the reconstituted nucleosomes in the AFM images using the method described in the Materials and Methods section indicated a gyration radius of $41 \pm 11 \mathrm{~nm}$ (mean \pm SD) for the nonacetylated nucleosome, but a radius of $57 \pm 13 \mathrm{~nm}$ for the acetylated nucleosome $(\mathrm{p}<0.001)$. The degree of saltinduced compaction of the acetylated and non-acetylated nucleosomes was compared in a buffer containing 100 or $200 \mathrm{mM} \mathrm{NaCl}$ by AFM imaging (Fig. 4D-G). Nonacetylated nucleosomes bound to each other and formed aggregates in the higher salt buffer whereas the acetylated chromatin exhibited an open, beads-on-a-string structure at this salt concentration. The minimum radius of a circle required to encompass an entire chromatin molecule was measured from these AFM images (Fig. $4 \mathrm{H}$ ). As the salt concentration increased, the average minimum radius of non-acetylated chromatin-but not that of acetylated chromatin-decreased. This difference may be caused by the increased electrical repulsion between nucleosomes contributed by the histone acetylation (see Discussion).

The addition of linker histone $\mathrm{H} 1$ into reconstituted chromatin is known to induce the formation of a fiber structure (Hizume et al., 2005). The width of the fiber is dynamic and changes depending on the buffer conditions. Thus, in $50 \mathrm{mM} \mathrm{NaCl}$, the fiber width is $20 \mathrm{~nm}$, but in $100 \mathrm{mM} \mathrm{NaCl}$ it is $30 \mathrm{~nm}$. As shown in Figures $5 \mathrm{~A}-\mathrm{H}$, the addition of histone $\mathrm{H} 1$ to either the non-acetylated or acetylated chromatin led to fiber formation. The efficiency of fiber formation was similar at different molar ratios of core histones:histone H1. However, the width of the fibers was dramatically decreased in the acetylated sample. In a buffer containing $100 \mathrm{mM}$ salt, the width of the nonacetylated fibers $(26.0 \pm 4.96 \mathrm{~nm}, \mathrm{n}=444$; Fig. $5 \mathrm{~K})$ was significantly greater than that of the acetylated fibers 
A

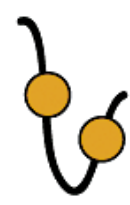

B

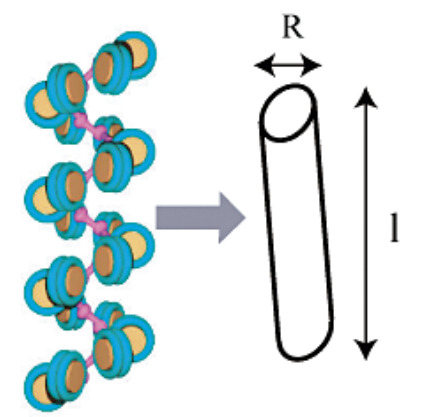

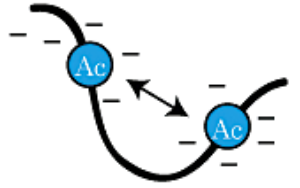

C

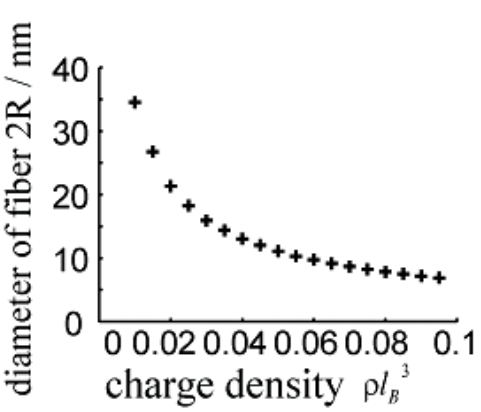

Fig. 6. Schematic models of the structural dynamics of the chromatin induced by histone acetylation. A: Potential effect of electric charge on the interaction between nucleosomes. The gyration radius of acetylated nucleosome was higher than that of non-acetylated nucleosomes, suggesting that the decrease in positive charge due to acetylation gives the nucleosome a more negative charge and produces a higher repulsive force between nucleosomes. B: The width of the H1-induced $30 \mathrm{~nm}$ fiber decreases depending on the degree of histone acetylation. C: Variation of fiber width with different charge densities.

(17.7 $\pm 5.14 \mathrm{~nm}, \mathrm{n}=439$; Fig. 5L) although this difference was hard to detect under conditions of $50 \mathrm{mM}$ salt (Fig. 5I, J).

\section{Discussion}

Histone-acetylation has been studied using genetic, biochemical, and cell biological analyses with a view towards understanding the mechanism by which histoneacetylation controls interactions between nuclear proteins and nucleosomes/chromatin. Proteins that contain a bromodomain, such as chromatin remodeling factors, histone acetyltransferases, and many transcription factors, have been shown to specifically interact with acetylated histones (Ornaghi et al., 1999; Jacobson et al., 2000; Shen et al., 2007). These studies suggest that specific proteins open the chromatin structure and lead to higher transcriptional activity. In this report, we have investigated the mechanism by which histone acetylation affects linker histone $\mathrm{H} 1$-induced fiber formation using both an in vitro reconstitution system (mediated by acetylated core histones) and native chromatin that is released from nuclei treated with TSA. Acetylation resulted in: 1) easy release of chromatin fibers out of nuclei, 2) a loss of salt-induced compaction in vitro, and 3 ) a decrease in the width of reconstituted H1-induced fibers.

\section{Two lines of evidence support the induction of chromatin decompaction by histone acetylation: inhibition of salt-dependent precipitation and reduction of the width of $\mathrm{H1}$-induced fibers}

The "higher-order" folding of a reconstituted nucleosome array has previously been detected as a salt-dependent precipitation of nucleosomal arrays (Garcia-Ramirez et al., 1995; Tse et al., 1998b; Shogren-Knaak et al., 2006). These studies have also shown that histone-acetylation inhibits the salt-dependent compaction of nucleosomes. Salt-dependent precipitation studies have raised the idea that the acetylation of specific lysine residues of histone tails is critical for chromatin decompaction. Indeed, using a native chemical ligation strategy to generate histone $\mathrm{H} 4$ acetylated at specific lysine residues, it has been shown that the acetylation of lysine 16 of histone $\mathrm{H} 4$ is sufficient to inhibit any salt-dependent compaction (ShogrenKnaak et al., 2006). The analysis of nucleosomal arrays, 
reconstituted from mutant core histones with lysine-toglutamine substitutions to mimic acetylation, showed that acetylation-mimics of $\mathrm{H} 2 \mathrm{~B}$ and $\mathrm{H} 4$ inhibit the saltdependent precipitation of nucleosomes (Wang and Hayes, 2008). In contrast to these previous studies, our study focused on the effect of hyper-acetylation on structural changes such as changes in the width of the H1induced fiber rather than on the sedimentation properties (or solubility) of the nucleosome array. Unexpectedly, our results showed that histone-acetylation did not prevent the formation of the H1-induced fiber. However, the fiber formed from hyper-acetylated nucleosomes was thinner than that formed from non-acetylated nucleosomes. It has been reported that histone $\mathrm{H} 1$ can bind to both hyperacetylated and non-acetylated chromatin but that it cannot induce $0.15 \mathrm{M} \mathrm{NaCl}$-dependent precipitation of an hyperacetylated nucleosome array (Ridsdale et al., 1990). This indicates that histone $\mathrm{H} 1$ induces "less folded state" chromatin with hyper-acetylated chromatin. The thinner fibers that we detected in this study could be fibers that exist in this lower folded state.

\section{Coarse-grained model of the decrease in the width of chromatin fibers due to acetylation}

To understand the mechanism by which acetylation induces a reduction in the width of the $\mathrm{H} 1$-induced fiber, it may be necessary to consider the physical properties of the fiber as a polymer. Since we did not examine the acetylation of specific lysine residues, the contribution of specific acetylation sites to the effects we observed is not clear. However, it is still possible to discuss the mechanism of structural change induced by acetylation in relation to the electrostatic properties of chromatin from a global point of view. DNA is a highly charged polymer that carries two negative elementary charges per bp. Core histones and $\mathrm{H} 1$ proteins are also charged polymers, carrying 147 and 54 positive elementary charges, respectively. One nucleosome forms from 147 bp of nucleosomal DNA, resulting in a total of 147 negative elementary charges per nucleosome. If this negatively charged nucleosome is acetylated on 22 lysine residues by $\mathrm{p} 300$, the number of charges per nucleosome increases to -169 e because acetylation of the histone tail neutralizes the positive charges of the lysines. This increase in negative charge should, in turn, increase the repulsing force between nucleosomes, so that the distance between nucleosomes increases, giving rise to the "beads-on-a-string" structure that was detected even under conditions of $200 \mathrm{mM}$ salt (Fig. 4G, 6A). This phenomenon that we observed is consistent with previous AFM studies that showed "cooperativity" (cooperative nucleosome occupation of a DNA fragment) of nucleosomes reconstituted on an $\sim 2 \mathrm{~kb}$ fragment as well as that interaction between neighboring nucleosomes was decreased by hyperacetylation (Yodh et al., 2002; Solis et al., 2007).

This electrostatic feature is important not only for nucleosome-nucleosome interaction but also for decompaction of the $30 \mathrm{~nm}$ fiber. EM observation of chromatin fibers purified from $\mathrm{NaBu}$-treated HeLa cells indicated that acetylated chromatin fibers were thinner than non-acetylated fibers (Annunziato et al., 1988). In our study, we also detected thinner fibers in both hyperacetylated nuclei and in reconstituted H1-induced fibers. Our in vitro experiments suggested that acetylation per se is directly responsible for making the fiber thinner, without the need for protein addition.

Acetylation of the lysine residues in core histones can locally adjust the charge of a chromatin component, a H1-containing nucleosome. A detailed model has been published (Arya and Schlick, 2009), in which the positional distribution of histone tails under different conditions such as the absence or presence of monovalent salts or of linker histones was analyzed and in which the effect of tail distribution on chromatin higher-order structure was simulated. In our present study, we found that the width of the chromatin fiber changed from 26.0 $\mathrm{nm}$ to $17.7 \mathrm{~nm}$ depending on the degree of acetylation in the histone tails. We considered this effect of hyperacetylation to correspond to a decrease in the remaining charge inside the fiber. By focusing on this decrease in fiber width caused by hyper-acetylation, we quantified the correlation between the change in the width of the fibers and the level of the charge that remains inside the fibers as follows: Since the H1-containing acetylated nucleosome contains more negative charges than the H1-containing non-acetylated nucleosome we considered a coarse-grained model to express the width of the chromatin fiber. Let us consider the fiber as a cylinder of radius $R$ and length $l$, with a total charge per unit volume of $-\rho \mathrm{e}$, and the solution conditions with ions as characterized by a Bjerrum length $l_{B} \equiv \mathrm{e}^{2} /$ $\varepsilon \mathrm{k}_{B} \mathrm{~T}$ ( $\varepsilon$ :dielectric constant of the solvent) and a Debye screening length $\kappa^{-1}=\left(8 \pi \mathrm{c}_{s} l_{B}\right)^{-1 / 2}\left(c_{s}\right.$ : salt concentration with $1: 1$ valences). The volume energy in the fiber per unit volume $\left(\mathrm{U}_{1}\right)$ excluding the Coulombic contribution would then be given as:

$$
U_{1}=-a
$$

where $a$ is a constant that represents the degree of attractive energy between nucleosomes. Consider that the destabilization energy, or surface energy, of the nucleosomes is located on the surface of the fiber since 
the nucleosomes on the surface of the fiber are not entirely surrounded by other nucleosomes. Accordingly, the surface energy of the cylinder per unit volume $\left(\mathrm{U}_{2}\right)$ is:

$$
U_{2}-2 \gamma / R \text {. }
$$

In this calculation the effect of the cylinder edge was neglected. When the fiber has no electronic charge, an infinite radius is possible. Because of the remaining negative charges, we consider the electrostatic energy $\left(\mathrm{U}_{3}\right)$ using the following equation:

$$
U_{3}=\frac{e^{2} \rho^{2}}{4 \varepsilon} R^{2}\left[\frac{1}{4}+\ln \left(\frac{\mathrm{R}+l_{D}}{R}\right)\right] .
$$

For the sake of simplicity the exact distribution of salts was neglected in the above equation.

The total energy of the fiber per unit volume (U) can then be approximated by the following equation:

$$
U=U_{1}+U_{2}+U_{3} .
$$

By minimizing the total energy $U$ with $R$, the width of the fiber is given as a function of the remaining charges (Fig. 6B, C). The more charges that remain, the wider the fiber becomes. This tendency is consistent with our experimental observations and suggests that, when a large amount of net charge remains, the fiber becomes unstable and tends to unfold into separate nucleosomal fibers without considering the role of small ions. The pair interaction between mononucleosomes has been shown to be complex; it has a minimum repulsive interaction at a concentration of $160 \mathrm{mM}$ sodium (Mangenot et al., 2002). It was also found that acetylated mononucleosomes induced a longer end-to-end distance of the linker DNA than non-acetylated mononucleosomes, indicating that acetylation causes an opening of the nucleosome structure (Toth et al., 2006). Unlike nucleosome core particles, the salt-concentration dependence of the pair potential of nucleosomes in a nucleosomal array (i.e. nucleosomes linked with each other by linker DNA) has been unclear although the dependence of the pair potential on the histone/DNA ratio has been reported (Nakai et al., 2005). This constitutes is a highly interesting topic for future study. However, in the absence of a detailed model, this particular trend regarding the width of the fiber can be roughly explained by a simple coarse-grained model. For a more detailed understanding of the structural effect of hyper-acetylation on chromatin fibers, a mesoscale structural simulation as previously described (Arya and Schlick, 2009) would be required.

\section{"Chromatin release" due to histone acetylation}

It is believed that chromosomes are partitioned in a well-organized manner in the nucleus in well defined three-dimensional territories (Cremer et al., 2006). One proposed model suggests that inactivated genes are located in condensed nuclear compartments. Conversely, activated genes are believed to be released into open areas called interchromatin compartments where they become accessible to the transcription machineries. Fluorescent microscopy studies have shown that HP1 dissociates from chromatin upon histone acetylation (Taddei et al., 2001; Maison et al., 2002) and that condensed chromatin regions are subsequently decondensed (Toth et al., 2004). Although this demonstrates that histone-acetylation affects chromatin organization in the nucleus, the detailed structural dynamics of chromatin fibers cannot be determined by fluorescence microscopy.

Chromatin fibers are more easily released from the nucleus after treatment with an HDAC inhibitor (Fig. 1). This result suggests that acetylated chromatin fibers are not "locked into" compact heterochromatin compartments. However, further experiments are required to conclude definitively that chromatin release is caused by histone-acetylation and not by a general weakening of nuclear integrity by HDAC inhibition. The resulting "detachment" of chromatin could facilitate gene relocation from the heterochromatin regions to the euchromatin regions. Our present observations of isolated nuclear chromatin suggest that this "detachment" is the most drastic structural change induced by histone acetylation. A combination of chromatin release from the condensed region, and specific binding between acetylated histones and proteins such as transcription factors, chromatin remodeling factors, and RNA polymerase complexes would therefore determine the status of "euchromatin."

\section{Acknowledgements}

We are grateful to Ms. Mami Kodama for her important contributions to all experiments in this study. We thank Mr. Matt Fagerburg and Ms. Jamie Gilmore for reading the manuscript.

\section{References}

Adkins NL, Watts M, Georgel PT: To the 30-nm chromatin fiber and beyond. Biochim Biophys Acta 1677: 12-23 (2004). 
Allfrey VG, Faulkner R, Mirsky AE: Acetylation and methylation of histones and their possible role in the regulation of Rna synthesis. Proc Natl Acad Sci USA 51: 786-794 (1964).

Annunziato AT, Frado LL, Seale RL, Woodcock CL: Treatment with sodium butyrate inhibits the complete condensation of interphase chromatin. Chromosoma 96: 132-138 (1988).

Arya G, Schlick T: A tale of tails: how histone tails mediate chromatin compaction in different salt and linker histone environments. J Phys Chem A 113: 40454059 (2009).

Balasubramanyam K, Swaminathan V, Ranganathan A, Kundu TK: Small molecule modulators of histone acetyltransferase p300. J Biol Chem 278: 19134-19140 (2003).

Belmont AS, Bruce K: Visualization of G1 chromosomes: a folded, twisted, supercoiled chromonema model of interphase chromatid structure. J Cell Biol 127: 287302 (1994).

Belyaeva E, Zhimulev I: RNA synthesis in the Drosophila melanogaster puffs. Cell Differ 4: 415-427 (1976).

Bustamante C, Zuccheri G, Leuba SH, Yang G, Samori B: Visualization and analysis of chromatin by scanning force microscopy. Methods 12: 73-83 (1997).

Cremer T, Cremer M, Dietzel S, Muller S, Solovei I, Fakan S: Chromosome territories--a functional nuclear landscape. Curr Opin Cell Biol 18: $307-316$ (2006).

Garcia-Ramirez M, Rocchini C, Ausio J: Modulation of chromatin folding by histone acetylation. J Biol Chem 270: 17923-17928 (1995).

Hancock R: A new look at the nuclear matrix. Chromosoma 109: 219-225 (2000).

Hizume K, Araki S, Yoshikawa K, Takeyasu K: Topoisomerase II, scaffold component, promotes chromatin compaction in vitro in a linker-histone H1dependent manner. Nucleic Acids Res 35: 2787-2799 (2007).

Hizume K, Yoshimura SH, Takeyasu K: Atomic force microscopy demonstrates a critical role of DNA superhelicity in nucleosome dynamics. Cell Biochem Biophys 40: 249-262 (2004).

Hizume K, Yoshimura SH, Takeyasu K: Linker histone $\mathrm{H} 1$ per se can induce three-dimensional folding of chromatin fiber. Biochemistry 44: 12978-12989 (2005).

Horn PJ, Peterson CL: Molecular biology: Chromatin higher order folding--wrapping up transcription. Science 297: 1824-1827 (2002).

Jacobson R, Ladurner A, King D, Tjian R: Structure and function of a human TAFII250 double bromodomain module. Science 288: 1422-1425 (2000).

Kadonaga JT: Eukaryotic transcription: an interlaced network of transcription factors and chromatinmodifying machines. Cell 92: 307-313 (1998).

Kim J, Yoshimura SH, Hizume K, Ohniwa RL, Ishihama A, Takeyasu K: Fundamental structural units of the Escherichia coli nucleoid revealed by atomic force microscopy. Nucleic Acids Res 32: 1982-1992 (2004).

Kobori T, Iwamoto S, Takeyasu K, Ohtani, T: Chromatin dynamics of unfolding and refolding controlled by the nucleosome repeat length and the linker and core histones. Biopolymers 85: 295-307 (2007).

Kobori T, Kodama M, Hizume K, Yoshimura SH, Ohtani T, Takeyasu K: Comparative structural biology of the genome: nano-scale imaging of single nucleus from different kingdoms reveals the common physicochemical property of chromatin with a $40 \mathrm{~nm}$ structural unit. J Electron Microsc (Tokyo) 55: 31-40 (2006).

Kornberg RD: Chromatin structure: a repeating unit of histones and DNA. Science 184: 868-871 (1974).

Luger K, Mader AW, Richmond RK, Sargent DF, Richmond TJ: Crystal structure of the nucleosome core particle at 2.8 A resolution. Nature 389: 251-260 (1997).

Maison C, Bailly D, Peters A, Quivy J, Roche D, Taddei A, Lachner M, Jenuwein T, Almouzni G: Higherorder structure in pericentric heterochromatin involves a distinct pattern of histone modification and an RNA component. Nat Genet 30: 329-334 (2002).

Mangenot S, Raspaud E, Tribet C, Belloni L, Livolant F: Interactions between isolated nucleosome core particles: A tail-bridging effect? Eur Phys J E 7: 221231 (2002).

Nakai T, Hizume K, Yoshimura SH, Takeyasu K, Yoshikawa K: Phase transition in reconstituted chromatin. Europhysi Lette 69: 1024-1030 (2005).

O'Neill T, Meersseman G, Pennings S, Bradbury E: Deposition of histone $\mathrm{H} 1$ onto reconstituted nucleosome arrays inhibits both initiation and elongation of transcripts by T7 RNA polymerase. Nucleic Acids Res 23: 1075-1082 (1995).

Ornaghi P, Ballario P, Lena A, González A, Filetici P: The bromodomain of Gen $5 p$ interacts in vitro with specific residues in the $\mathrm{N}$ terminus of histone $\mathrm{H} 4$. J Mol Biol 287: 1-7 (1999).

Ridsdale JA, Hendzel MJ, Delcuve GP, Davie JR: Histone acetylation alters the capacity of the $\mathrm{H} 1$ histones to condense transcriptionally active/competent chromatin. J Biol Chem 265: 5150-5156 (1990).

Robinson PJ, Rhodes D: Structure of the '30 nm' chromatin fibre: a key role for the linker histone. Curr Opin Struct Biol 16: 336-343 (2006).

Schneider SW, Larmer J, Henderson RM, Oberleithner 
$\mathrm{H}$ : Molecular weights of individual proteins correlate with molecular volumes measured by atomic force microscopy. Pflugers Arch 435: 362-367 (1998).

Shen W, Xu C, Huang W, Zhang J, Carlson J, Tu X, Wu J, Shi Y: Solution structure of human Brg1 bromodomain and its specific binding to acetylated histone tails. Biochemistry 46: 2100-2110 (2007).

Shogren-Knaak M, Ishii H, Sun JM, Pazin MJ, Davie JR, Peterson CL: Histone H4-K16 acetylation controls chromatin structure and protein interactions. Science 311: 844-847 (2006).

Simon RH, Felsenfeld, G: A new procedure for purifying histone pairs $\mathrm{H} 2 \mathrm{~A}+\mathrm{H} 2 \mathrm{~B}$ and $\mathrm{H} 3+\mathrm{H} 4$ from chromatin using hydroxylapatite. Nucleic Acids Res 6: 689-696 (1979).

Solis FJ, Bash R, Wang H, Yodh J, Lindsay SA, Lohr D: Properties of nucleosomes in acetylated mouse mammary tumor virus versus $5 \mathrm{~S}$ arrays. Biochemistry 46: 5623-5634 (2007).

Stalder J, Larsen A, Engel JD, Dolan M, Groudine M, Weintraub H: Tissue-specific DNA cleavages in the globin chromatin domain introduced by DNAase I. Cell 20: 451-460 (1980).

Strahl BD, Allis CD: The language of covalent histone modifications. Nature 403: 41-45 (2000).

Taddei A, Maison C, Roche D, Almouzni G: Reversible disruption of pericentric heterochromatin and centromere function by inhibiting deacetylases. Nat Cell Biol 3: 114-120 (2001).

Thoma F, Koller T: Influence of histone H1 on chromatin structure. Cell 12: 101-107 (1977).

Toth K, Brun N, Langowski J: Chromatin compaction at the mononucleosome level. Biochemistry 45: 15911598 (2006).

Toth KF, Knoch TA, Wachsmuth M, Frank-Stohr M, Stohr M, Bacher CP, Muller G, Rippe K: Trichostatin
A-induced histone acetylation causes decondensation of interphase chromatin. J Cell Sci 117: 4277-4287 (2004).

Tse C, Georgieva EI, Ruiz-Garcia AB, Sendra R, Hansen JC: Gcn5p, a transcription-related histone acetyltransferase, acetylates nucleosomes and folded nucleosomal arrays in the absence of other protein subunits. J Biol Chem 273: 32388-32392 (1998a).

Tse C, Sera T, Wolffe AP, Hansen JC: Disruption of higher-order folding by core histone acetylation dramatically enhances transcription of nucleosomal arrays by RNA polymerase III. Mol Cell Biol 18: 46294638 (1998b).

Wang X, Hayes JJ: Acetylation mimics within individual core histone tail domains indicate distinct roles in regulating the stability of higher-order chromatin structure. Mol Cell Biol 28: 227-236 (2008).

Weintraub $\mathrm{H}$ : Histone-H1-dependent chromatin superstructures and the suppression of gene activity. Cell 38: 17-27 (1984).

Weintraub H, Groudine M: Chromosomal subunits in active genes have an altered conformation. Science 193: 848-856 (1976).

Yodh JG, Woodbury N, Shlyakhtenko LS, Lyubchenko YL, Lohr D: Mapping nucleosome locations on the 208-12 by AFM provides clear evidence for cooperativity in array occupation. Biochemistry 41: 3565-3574 (2002).

Zhang W, Bone JR, Edmondson DG, Turner BM, Roth SY: Essential and redundant functions of histone acetylation revealed by mutation of target lysines and loss of the Gcn5p acetyltransferase. EMBO J 17: 3155 3167 (1998).

Zweidler A: Resolution of histones by polyacrylamide gel electrophoresis in presence of nonionic detergents. Methods Cell Biol 17: 223-233 (1978). 